\title{
Plasmon-enhanced nanosoldering of silver nanoparticles for high-conductive nanowires electrodes
}

\author{
Yuan-Yuan Zhao ${ }^{1, \dagger}$, Xue-Liang Ren ${ }^{2, \dagger}$, Mei-Ling Zheng $2,3 *$, Feng Jin², \\ Jie Liu², Xian-Zi Dong ${ }^{2}$, Zhen-Sheng Zhao ${ }^{2}$ and Xuan-Ming Duan ${ }^{1 *}$
}

The silver nanowires (Ag NWs) electrodes, which consist of incompact Ag nanoparticles (NPs) formed by multi-photon photoreduction, usually have poor conductivities. An effective strategy for enhancing conductivity of the Ag NWs electrodes is plasmon-enhanced nanosoldering (PLNS) by laser irradiation. Here, plasmon-enhanced photothermal effect is used to locally solder Ag NPs and then aggregates of these NPs grow into large irregular particles in PLNS process. Finite element method (FEM) simulations indicate that the soldering process is triggered by localized surface plasmon-induced electric field enhancement at "hot-spots". The effectiveness of PLNS for enhancing conductivity depends on laser power density and irradiation time. By optimizing the conditions of PLNS, the electrical conductivity of Ag NWs is significantly enhanced and the conductivity $\sigma_{\mathrm{s}}$ is increased to $2.45 \times 10^{7} \mathrm{~S} / \mathrm{m}$, which is about $39 \%$ of the bulk Ag. This PLNS of Ag NWs provides an efficient and cost-effective technique to rapidly produce large-area metal nanowire electrodes and capacitors with high conductivity, excellent uniformity, and good flexibility.

Keywords: silver nanowires; silver nanoparticles; direct laser writing; laser nanosoldering; photothermal effect; conductivity

Zhao YY, Ren XL, Zheng ML, Jin F, Liu J et al. Plasmon-enhanced nanosoldering of silver nanoparticles for high-conductive nanowires electrodes. Opto-Electron Adv 4, 200101 (2021).

\section{Introduction}

Silver nanowire (Ag NWs) electrodes, with accurate controllable resistance $R$ via tuning geometry, have received much attention as the crucial components in various electronic devices, such as flexible circuits ${ }^{1-4}$, organic solar cells $s^{5,6}$, and touch panels ${ }^{7-9}$. The common methods of patterning Ag NWs electrodes is photolithography, inkjet or printed technology ${ }^{10-13}$. In particular, femto- second laser direct writing (FsLDW), including multiphoton absorption induced photoreduction or photodynamic assembly of silver nanoparticles (Ag NPs), is used to build Ag NWs for engineered patterns in two and three dimensions with submicron resolution ${ }^{14-17}$. This method is widely used in the preparation of various optoelectronic devices ${ }^{14,16,18,19}$. However, the reported Ag NWs built by the FsLDW are all composed of aggregates

${ }^{1}$ Guangdong Provincial Key Laboratory of Optical Fiber Sensing and Communications, Institute of Photonics Technology, Jinan University, Guangzhou 511443, China; 'Laboratory of Organic NanoPhotonics and CAS Key Laboratory of Bio-Inspired Materials and Interfacial Science, Technical Institute of Physics and Chemistry, Chinese Academy of Sciences, Beijing 100190, China; ${ }^{3}$ School of Future Technologies, University of Chinese Academy of Sciences, Yanqihu Campus, Beijing 101407, China.

tThese authors contributed equally to this work.

"Correspondence: ML Zheng, E-mail: zhengmeiling@mail.ipc.ac.cn; XM Duan, E-mail: xmduan@jnu.edu.cn

Received: 31 December 2020; Accepted: 10 February 2021; Published online: 25 December 2021

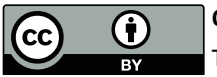

Open Access This article is licensed under a Creative Commons Attribution 4.0 International License

To view a copy of this license, visit http://creativecommons.org/licenses/by/4.0/.

(C) The Author(s) 2021. Published by Institute of Optics and Electronics, Chinese Academy of Sciences. 
of much smaller Ag NPs with void or polymer inclusions ${ }^{15,16}$, which leads to poor conductivity. In our previous study, we have systematically fabricated the patterned Ag NWs electrodes with the designed resistance by FsLDW ${ }^{14,15}$. The highest value of electrical conductivity $\sigma_{\mathrm{s}}$ is $2.44 \times 10^{6} \mathrm{~S} / \mathrm{m}$, which is about $3.9 \%$ of bulk $\mathrm{Ag}^{15}$. To our knowledge, most of the resistance $R$ of Ag NWs comes from slight surface oxidation and particle-particle gaps of Ag NPs compared to bulk Ag. To enhance the conductivity $\sigma_{\mathrm{s}}$ and reduce its resistance $R$, we must minimize the energy dissipation of conduction electrons in Ag NWs electrodes by reducing the gaps and increasing the contact areas among the NPs ${ }^{16}$.

The widely applied methods to increase the contact areas of adjacent Ag NPs and enhance electrical conductivity of Ag NWs electrodes are thermal and laser annealing $^{20-23}$. Jahn et al. described the utilization of thermal treatment for ink-jetting Ag patterns on glass and flexible substrates, and the conductivity $\sigma_{\mathrm{s}}$ is increased to $43 \%$ and $18 \%$ of bulk $\mathrm{Ag}$, respectively ${ }^{24}$. Nonetheless, a temperature of one hundred Celsius degrees is required during thermal annealing, leading to the unacceptable damage of the substrates ${ }^{25,26}$. Laser annealing, as an alternative method, has been systematically investigated in recent years, benefiting from the efficient energy deposition and reduced heat affected zone, which would allow

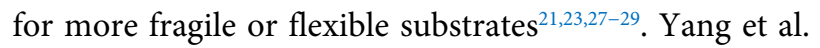
demonstrated the rapid laser irradiation to anneal the printed Ag NPs film, and the conductivity $\sigma_{\mathrm{s}}$ is $4 \%-8.3 \%$ of bulk $\mathrm{Ag}^{30}$. Garnett et al. reported a light-induced plasmonic-nanowelding of Ag NWs technology, similar to laser annealing which significantly improves the conductivity of the connected Ag NWs networks ${ }^{23}$. After the light illumination, the conductivity is roughly three orders of magnitude higher than the previous results. Considering the poor conductivity of $\mathrm{Ag}$ NWs electrodes consist of incompact NPs fabricated by FsLDW methods, the above laser annealing can be used to heat Ag NWs electrodes locally at the gaps of connected region to solder the incompact $\mathrm{Ag} \mathrm{NPs}$ and improve its conductivity.

In this study, we propose an optical method for enhancing the electric conductivity of the Ag NWs by plasmon-enhanced laser nanosoldering (PLNS). PLNS of Ag NWs utilizes the plasmon-enhanced photothermal effect to realize the localized nanosoldering at room temperature. We have investigated the effects of two important parameters, including laser power density and nanosol- dering time on the electric conductivity of Ag NWs. We found that the resistance of Ag NWs is reduced significantly through either increasing the laser power density or the nanosoldering time. After the plasmon-enhanced laser nanosoldering, the obtained Ag NWs have a maximal electric conductivity of $2.45 \times 10^{7} \mathrm{~S} / \mathrm{m}$ at the proline concentration of $0.10 \mathrm{M}$, the laser power density of 9.55 $\mathrm{MW} / \mathrm{cm}^{2}$ and the nanosoldering time of 15 minutes. This study provides an efficient and cost-effective approach for enhancing electrical conductivity of Ag NWs in a controllable fashion, and promoting direct writing $\mathrm{Ag}$ NWs for further use as active SERS substrates, transparent electrode, capacitor, light-emitting diodes, thin-film solar cells, etc.

\section{Results and discussion}

\section{Experimental design for plasmon-enhanced laser nanosoldering of Ag NWs}

The schematic illustrations of the plasmon-enhanced laser nanosoldering system and the detailed optical experimental setup are presented in Fig. 1(a). Pulsed Nd:YAG laser (Spectra-Physics, Quanta-Ray) with a center wavelength of $532 \mathrm{~nm}$, pulse width of $8 \mathrm{~ns}$ and repetition rate of $10 \mathrm{~Hz}$, is used as a light source for laser nanosoldering. Ag NWs were fabricated by FsLDW and showed comparatively high absorption at this wavelength ${ }^{6,7}$. The nanosecond(ns)-pulse laser beam passes through a pinhole to form a light spot with $5 \mathrm{~mm}$ diameter, in order to shape the laser beam to produce a uniform radiation optical field. The attenuator is employed to adjust the laser power density, and a mechanical shutter is utilized to control the laser irradiation time as accurate as $1 \mathrm{~ms}$, in order to investigate the influence of laser irradiation on the electric conductivity.

The Ag NWs with Ag NPs at an average size of $~ 30$ $\mathrm{nm}$ were fabricated by FsLDW as shown in Fig. 1(b), based on the multi-photon absorption induced photoreduction of $\mathrm{Ag}$ ions ${ }^{15}$. In our PLNS process, the absorption of $n s$-pulse lasers for Ag NPs induces rapid heating and melting at time scale of a few picoseconds ${ }^{31-34}$. Thus, the adjacent Ag NPs are in contact and welded together after the laser irradiation. This photothermal effect can be strongly enhanced when several NPs aggregate together ${ }^{35-44}$, arising from surface plasmon resonance (SPR) of Ag NPs. Thus, a low density of light concentrates at the gaps between two adjacent Ag NPs, and these areas are referred to as "hot-spots" 23,36-44. 
Interaction of light with $\mathrm{Ag}$ NPs is modeled using the previously reported method ${ }^{36,37}$, and the numerical calculations of plasmon-enhanced electric field are shown in Fig. 1(c). The electric field among the particles is significantly enhanced when the polarization direction of the incident light is parallel to the interparticle axis instead of perpendicular to the interparticle axis. The heating values of Ag NPs are proportional to the local light field intensity $^{34-36}$. The enhanced light intensity is beneficial to increase the local nanosoldering temperature to the melting point in room temperature environment.

Figure 1(d) shows the schematic illustrations of the plasmon-enhanced laser nanosoldering process. Ag NWs electrodes consisting of Ag NPs are placed on a cover glass by the irradiation with a $532 \mathrm{~nm}$ laser. The aggregation of Ag NPs leads to the formation of Ag NWs. Thus, the fabricated Ag NWs electrodes are incompact, as shown in Fig. 1(d-i). Under the laser irradiation, Ag NWs have a strong optical absorption at $532 \mathrm{~nm}$ owing to SPR absorption. This photothermal effect can make the nanoparticles generate temperature enhancement through converting light energy into thermal energy. As the temperature rises, the melting or sintering occurs on the surface of nanoparticles, and is enhanced at the particle-particle gaps by plasmon-enhanced light field shown in Fig. 1(d-ii). As the nanosoldering time increases, sintering occurs by atoms migrating mainly along the particle surface ${ }^{42}$. When two nanoparticles join together, they create a "neck". The melting and sintering process of Ag nanoparticles begins with rapid "neck" formation followed by "neck" growth driven by surface diffusion $^{42-44}$. Finally, the recrystallization of Ag NPs leads to the formation of compact Ag NWs as shown in Fig. 1(d-iii).

\section{Morphology characterization of Ag NWs electrodes} Firstly, we demonstrate the effect of PLNS process to modify the morphology of Ag NWs. We prepare the Ag NWs by the previously reported FsLDW ${ }^{14,15}$. The surface topographies, such as shape and roughness, play important roles in the electrical properties of the Ag NWs electrodes. The average widths of $\mathrm{Ag}$ nanowires can be controlled within the range of $500 \mathrm{~nm}$ with the input laser power of $0.2 \mathrm{~mW}$ and the laser scanning speed of $6 \mu \mathrm{m} / \mathrm{s}$ as shown in Fig. 1(e). Before the laser nanosoldering, the Ag NWs consist of individual Ag NPs, and a large number of nano-intervals exist among the nano-particles as shown in Fig. 1(e-i). After the laser nanosoldering, the
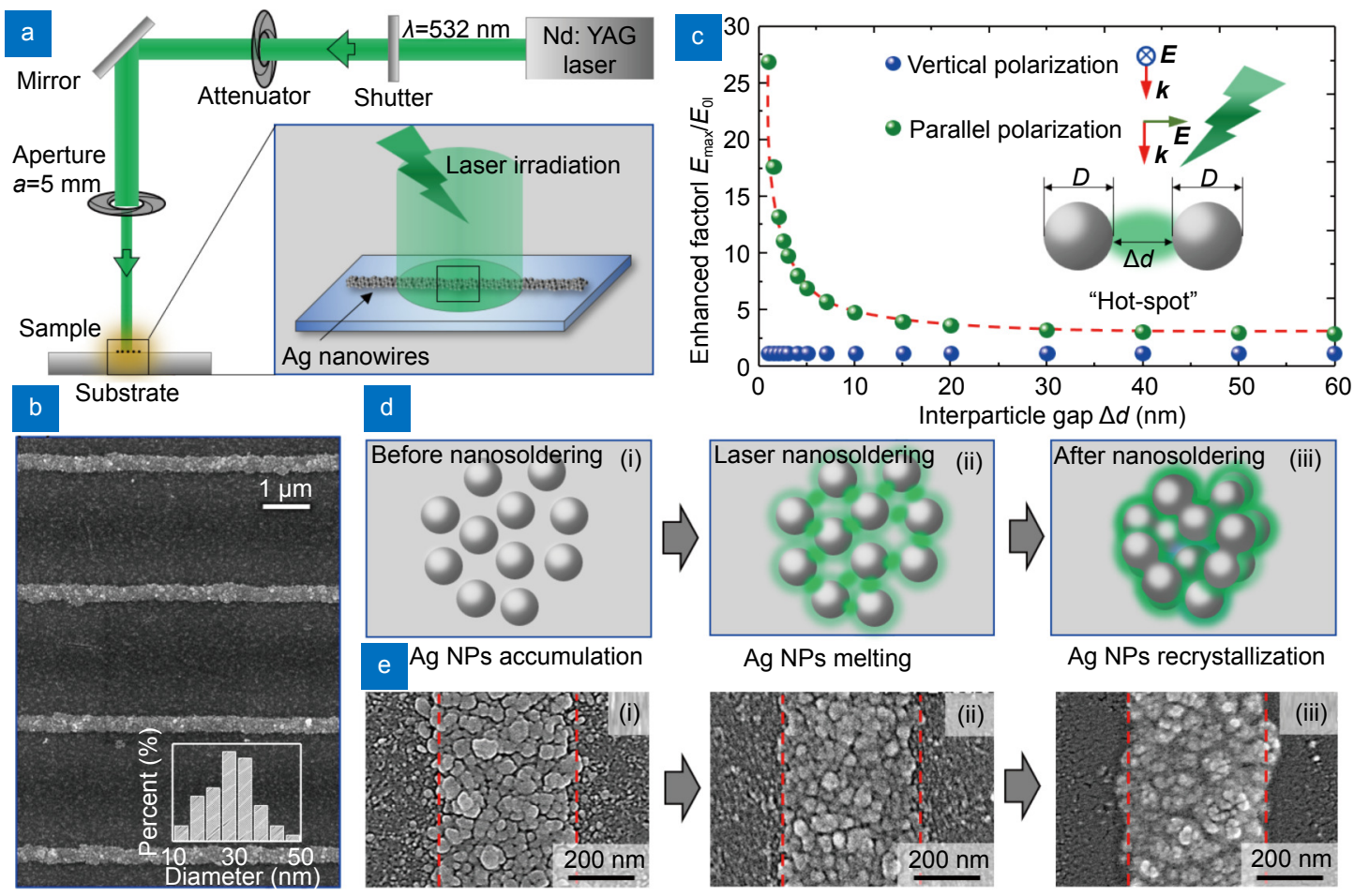

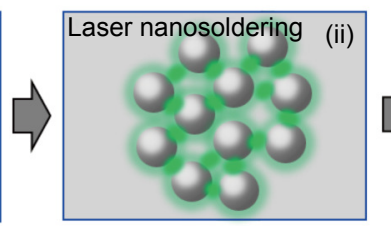

Ag NPs melting
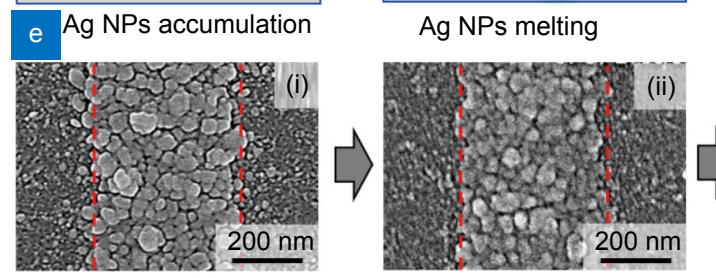

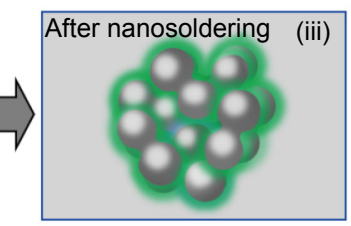

Ag NPs recrystallization

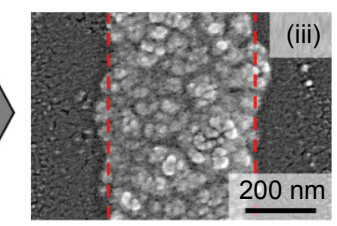

Fig. 1 | (a) Schematic of experimental system for PLNS. (b) Scanning electron microscope (SEM) image of Ag NWs with inset showing the size distribution of Ag NPs in Ag NWs. (c) Plasmon-enhanced electric field as a function of interparticle gap for light polarization direction parallel and vertical to the interparticle axis. (d) Schematic illustration of PLNS with increasing laser irradiation time. (e) SEM images of the morphological changes of Ag NWs in PLNS process. 
melting of nanoparticles leads to the reduction of nanointervals and the increase of particle size as shown in Fig. 1(e-ii). With the increase of nanosoldering time, the melting is more sufficient, and the nanoparticles are tightly connected as shown in Fig. 1(e-iii). The PLNS process can accelerate the accumulation and agglomeration of Ag NPs in the region exposed to sufficient laser power input, thus forming the relatively smooth and compact continuous NWs.

In order to further demonstrate the changes in the aggregation morphology of nanoparticles before and after the nanosoldering, we draw a comparison map about the transmission electron microscopy (TEM) of Ag NWs on carbon-coated copper grids with or without the ns-pulse laser irradiation. As shown in Fig. 2(f)-2(h), Ag NPs are sintered and welded together to form neck between the particle-particle gaps in a programmed manner by irradiating laser pulses of $532 \mathrm{~nm}$ and $9.55 \mathrm{MW} / \mathrm{cm}^{2}$ for 15 min, which are different from the isolated ones shown in Fig. 2(a)-2(c). Therefore, PLNS changes the aggregation morphology of the nanoparticles, but not the lattice distribution at the atomic level. Laser pulses were utilized to join, to hold closely, and to solder Ag NPs on TEM grids.

Further evidence of this mechanism comes from the magnified HRTEM images and the selected area electron diffraction (SAED) patterns of Ag NWs before and after the PLNS process [Fig. 2(d,i) and 2(e, g), respectively]. Lattice-resolved HRTEM images taken on the individual Ag NP [Fig. 2(d, i)] in single nanowire [Fig. 2(a, f)] confirmed that two typical crystal orientations [111] and [200] existed at the Ag NP and they were the polycrystalline whether or not being illuminated. Moreover, SAED patterns collected for representative Ag NPs before and after the nanosoldering process [Fig. 2(e) and 2(j), respectively] also prove that the basic constituent of Ag NP is polycrystalline. This comparison suggests that individual Ag NP does not completely melt to form a single crystal during the laser nanosoldering, but melt and recrystallize at the interface among adjacent Ag NPs after the illumination. PLNS process promotes the aggregation of small Ag NPs to form larger particles, and further makes the nanoparticles welded together to form dense nanowires.

\section{Electrical properties of Ag NWs electrodes}

Laser nanosoldering results in the appearance of large nanoparticles due to the laser-induced melting and coalescence. Meanwhile, laser nanosoldering enhances the neck growth between particles and therefore the electron diffusion coefficient correspondingly reduces with the decrease of the defect after photothermal sintering and welding ${ }^{11,21,42}$. Therefore, laser nanosoldering
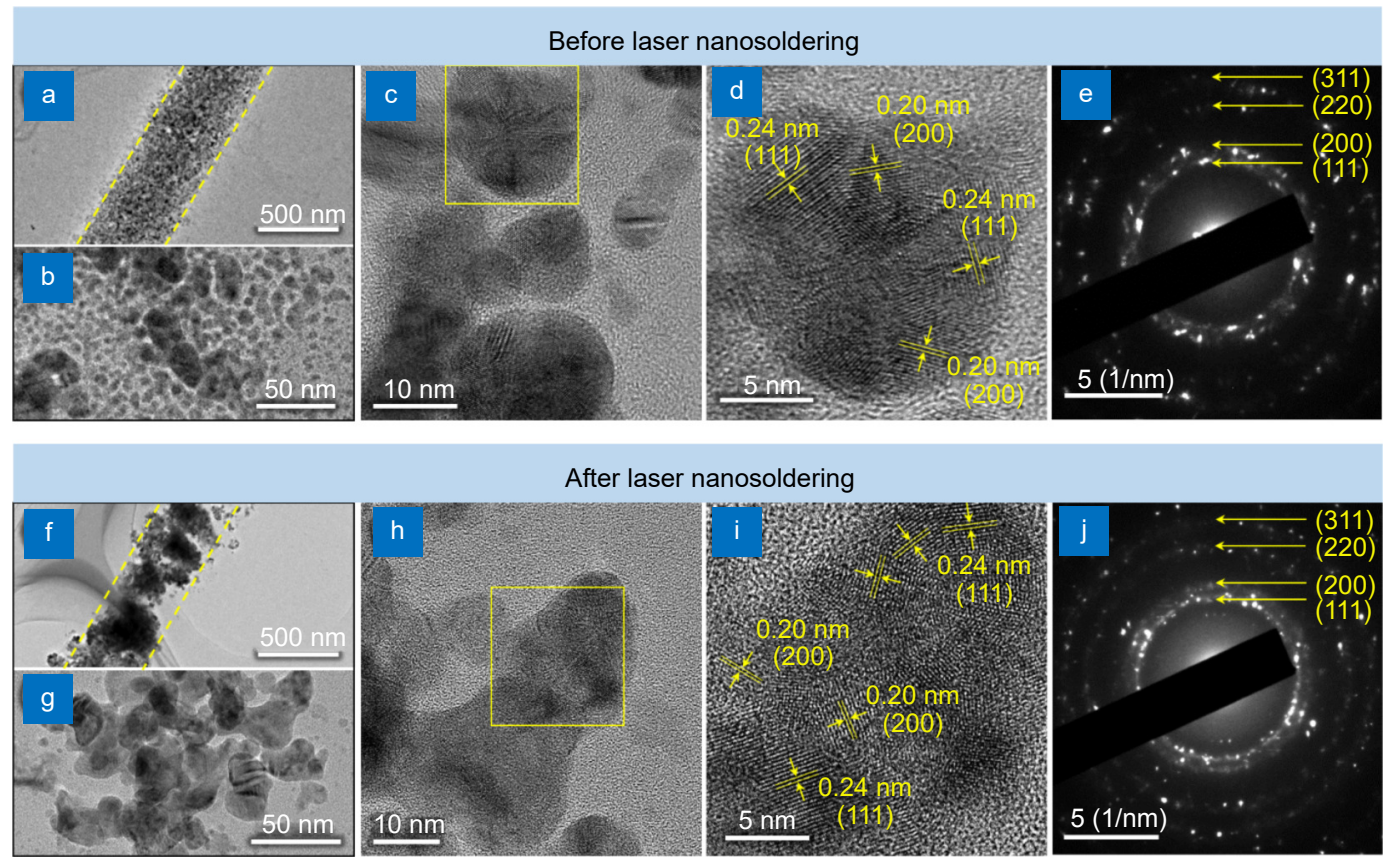

Fig. 2 | TEM images of Ag NWs for overall (a), local (b) and magnified (c) topographies before the laser illumination. (d) Typical HRTEM images and (e) SAED patterns of Ag NPs before the laser illumination. TEM images of Ag NWs for overall (f), local (g) and magnified (h) topographies after the illumination. (i) Typical HRTEM images and (j) SAED pattern of Ag NPs after laser illumination for 15 min. 
significantly affects the electrical properties of Ag NWs structured by Ag NPs. The influence of laser nanosoldering on the electric conductivity of Ag NWs has been investigated first. We compared the conductivity of nanowires before and after the laser nanosoldering under the experimental conditions of the irradiating laser power density of $9.55 \mathrm{MW} / \mathrm{cm}^{2}$ and the nanosoldering time of $15 \mathrm{~min}$.

The resistance of the Ag NWs is measured using a two-probe measurement method [Fig. 3(a)] by depositing the Ag film electrodes on two sides of the Ag NWs (Fig. S1). The typical $I-V$ curves of Ag NWs before and after the laser nanosoldering are illustrated in Fig. 3(b). The three-dimensional morphology of Ag NWs obtained by ion beam cutting before and after the laser nanosoldering shown in Fig. 3(c, e) are used as a support proof to assist in the analyses of electrical properties. Figure $3(\mathrm{~d}, \mathrm{f})$ show the corresponding AFM images and the profiles of cross section. The fabricated Ag NWs with the average linewidth of $847 \mathrm{~nm}$, is consisted of $28 \mathrm{~nm}$-size NPs before the nanosoldering ${ }^{15}$. It can be seen that the surface of the Ag NWs is relative smooth due to the component of small NPs, but the roughness is increased since small NPs welded together to form larger NPs after the laser nanosoldering. The height of the Ag NWs increases from $60 \mathrm{~nm}$ to $120 \mathrm{~nm}$ and the cross-sectional area is about $0.02682 \mu \mathrm{m}^{2}$. The length of Ag NWs for electrical characterization is $50 \mu \mathrm{m}$. On the basis of Ohm's law, the electric resistivity of Ag NWs $\left(\rho_{\mathrm{s}}\right)$ can be written as follows ${ }^{15,16}$ :

$$
\rho_{s}=R \cdot \frac{A}{L}=R \cdot \frac{d h}{2 L},
$$

where $A, R, d, h$ and $L$ represent the cross-sectional area, resistance, linewidth, height and length of Ag NWs, respectively. The electric conductivity $\sigma_{\mathrm{s}}=1 / \rho_{\mathrm{s}}$ has been enhanced corresponding to the resistance from $2.44 \times 10^{6}$ $\mathrm{S} / \mathrm{m}$ to $24.51 \times 10^{6} \mathrm{~S} / \mathrm{m}$, which is up to $39 \%$ of bulk $\mathrm{Ag}$ $\left(63 \times 10^{6} \mathrm{~S} / \mathrm{m}\right)$.

The control of irradiation power and time is critical to achieve optimal nanosoldering. In our experiment, we have varied the average nanosoldering laser power $(P)$ of $2,5,8,11$ and $15 \mathrm{~mW}$, which is equivalent to the power density $\left(I_{0}\right)$ of $1.27,3.18,5.10,7.01$ and $9.55 \mathrm{MW} / \mathrm{cm}^{2}$. For $n s$-pulse laser, the power density $\left(I_{0}\right)$ can be calculated by the formula $I_{0}=P /\left(\pi \omega^{2} \times \tau f\right)$, where $P$ is the average laser power, $\tau$ is pulse width, $f$ is repetition frequency and $\omega$ is radius of the beam. Figure 4(a) shows the experimental dependence of the resistance $R$ on laser power density $I_{0}$ for single Ag NWs electrode under the fixed nanosoldering time of $11 \mathrm{~min}$. With the increasing of the laser power density $I_{0}$, the resistance $R$ decreases significantly. This is because the conductivity behavior relies on the aggregation degree and size of the Ag NPs. Furthermore, the dependency between the resistance $R$ and the laser nanosoldering time has also been

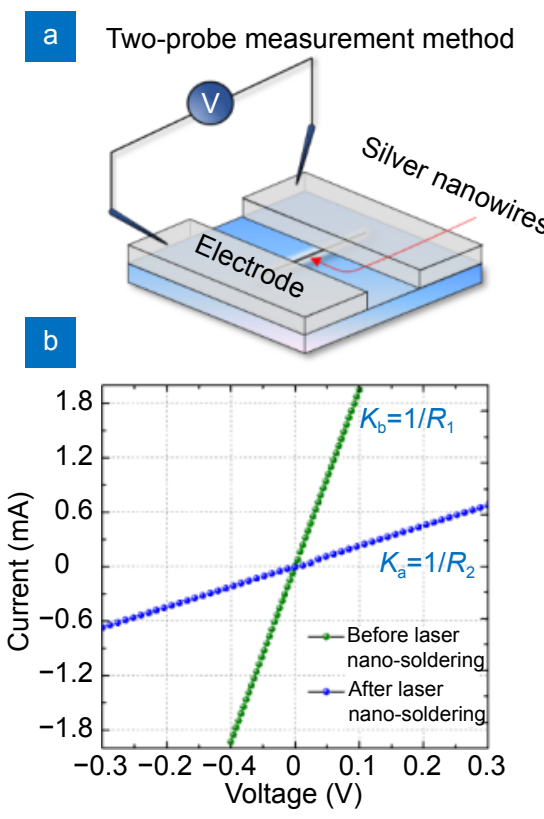

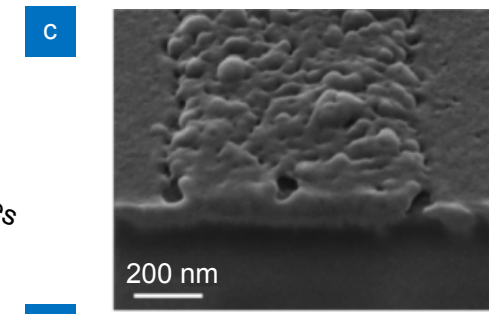
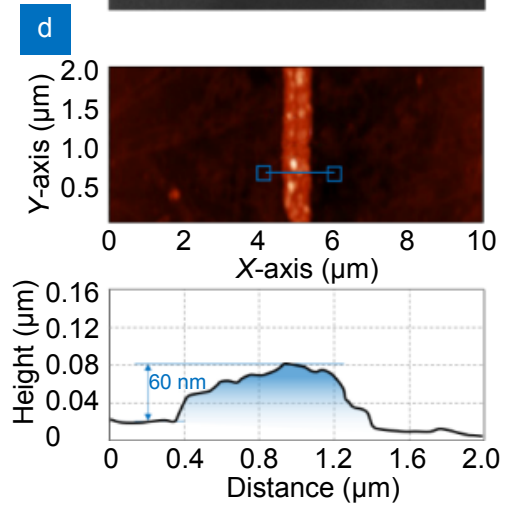
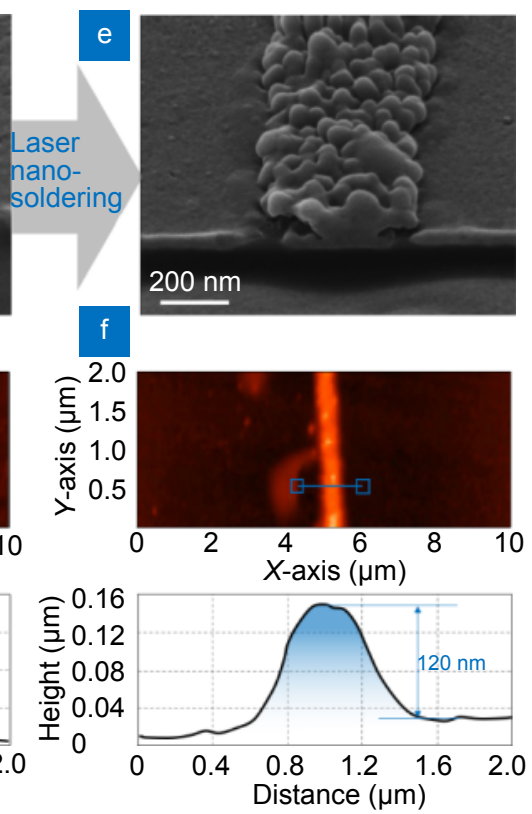

Fig. 3 | (a) Schematic of two-probe measurement method. (b) I-V curve of the fabricated Ag NWs before and after the laser nanosoldering. (c, f) Morphology of Ag NWs cut by focus ion beam. (d, $\mathbf{g})$ AFM images and the height profile of the Ag NWs before and after the laser nanosoldering. 

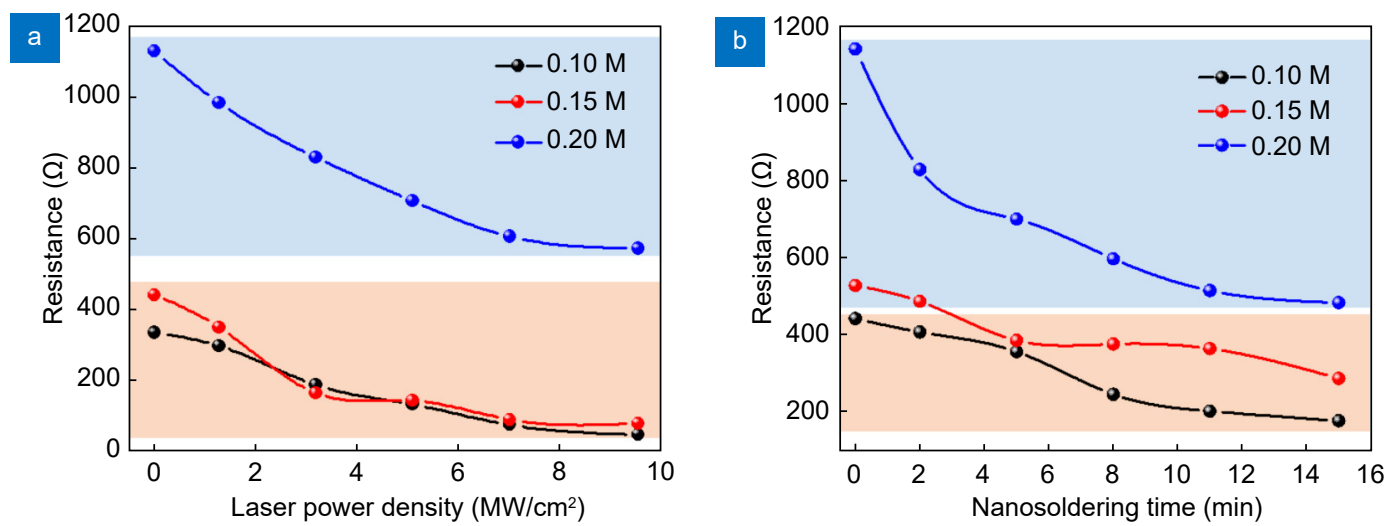

Fig. 4 | (a) Measured resistance of the Ag NWs electrodes as a function of the laser nanosoldering power density with the laser nanosoldering time of $11 \mathrm{~min}$. (b) Measured resistance of the Ag NWs electrodes as a function of the laser nanosoldering time with laser nanosoldering power density of $7.01 \mathrm{MW} / \mathrm{cm}^{2}$.

investigated as shown in Fig. 4(b). The resistance of Ag NWs is declined with the increasing of nanosoldering time when the concentration of proline is $0.10 \mathrm{M}$ and the laser power density of $7.01 \mathrm{MW} / \mathrm{cm}^{2}$. The resistance of Ag NWs declined from $445 \Omega$ to $180 \Omega$ after the laser nanosoldering. With $0.10 \mathrm{M}$ or $0.15 \mathrm{M}$ proline presents in the $\mathrm{Ag}$ precursor solutions, the resistance of $\mathrm{Ag} \mathrm{NWs}$ exhibits similar variation tendency and similar resistance range due to the similar particle's size distribution. As shown in Figs. 5(a) and S2, the average size of the Ag NPs is about $30 \mathrm{~nm}, 28 \mathrm{~nm}$ and $15 \mathrm{~nm}$ for different concentrations of prolines of $0.10 \mathrm{M}, 0.15 \mathrm{M}$ and $0.20 \mathrm{M}$, respectively. All of the Ag NWs fabricated in Ag ions solution with different concentrations of proline have a similar variation tendency between the resistance $R$ and laser power density as well as laser nanosoldering time, see the experimental results in Figs. S3 and S4. The increase in conductivity of Ag NWs approaches saturation state with the increasing of either laser power density or the nanosoldering time.

\section{Mechanism analysis of PLNS for Ag nanowires}

In PLNS process, Ag NP is considered to absorb many photons of pulsed lasers successively and reaches the temperature as high as the melting point ${ }^{32,33}$. Thus, the adjacent Ag NPs are welded together after multiple pulses laser irradiation. This PLNS process increases the conductivity of Ag NWs containing Ag NPs. Currently, the heating behavior among individual NP has been well established ${ }^{32-34}$. In the steady-state regime, the local temperature increasing $\Delta T\left({ }^{\circ} \mathrm{C}\right)$ around a single NP is described by the following equation:

$$
\begin{aligned}
& \Delta T(r)=\frac{V_{\mathrm{NP}} Q}{4 \pi k_{0} r}, \\
& Q=\frac{\omega}{8 \pi} E_{0}^{2}\left|\frac{3 \varepsilon_{0}}{2 \varepsilon_{0}+\varepsilon_{\mathrm{NP}}}\right|^{2} \operatorname{Im}\left(\varepsilon_{\mathrm{NP}}\right),\left(r \geq R_{\mathrm{NP}}\right),
\end{aligned}
$$

where $r$ is the distance from the center of a NP, $k_{0}$ is the thermal conductivity of the surrounding medium, and $V_{\mathrm{NP}}$ is the NP volume. The temperature increment of all particles depends on the size of the particles and the laser pulse power ${ }^{33}$. The above model only describes the nanosoldering behaviors for individual NP. However, Ag NWs structures with different aggregation morphologies are rarely considered in the studies of the nanosoldering behaviors. In our study, Ag NWs fabricated by FsLDW are composed of the aggregation of NPs. Laser nanosoldering for Ag NWs can be strongly enhanced because several NPs aggregate together form the "hot-spots" $36-43$, as shown in Fig. 5(b). Whereas grain growth requires the temperature approaching the melting point $\left(T_{\mathrm{m}}\right)$ in bulk materials melting, however, it can occur at temperatures as low as $\sim 0.2 T_{\mathrm{m}}$ (see Fig. S5) in Ag NPs aggregations ${ }^{45}$. Thus, local melting can occur and form necks at the interfaces, enhancing electrical conductivity of Ag NWs structured by Ag NPs.

We have investigated the interaction between nanoparticles and light using commercially available finite element method (FEM) solver COMSOL Multiphysics. The surface plasmon resonances are usually dependent on the size, shape, and degree of particle-to-particle coupling $^{35-43}$. The spatial distribution of Ag NPs is irregular and random but the particle's size meets the normal distribution in Fig. 5(a). In our simulation model, the aggregate states of several NPs are arranged via a statistical method according to the experimental results in Fig. 5(a). A random system of Ag NPs has "hot-spots" where 

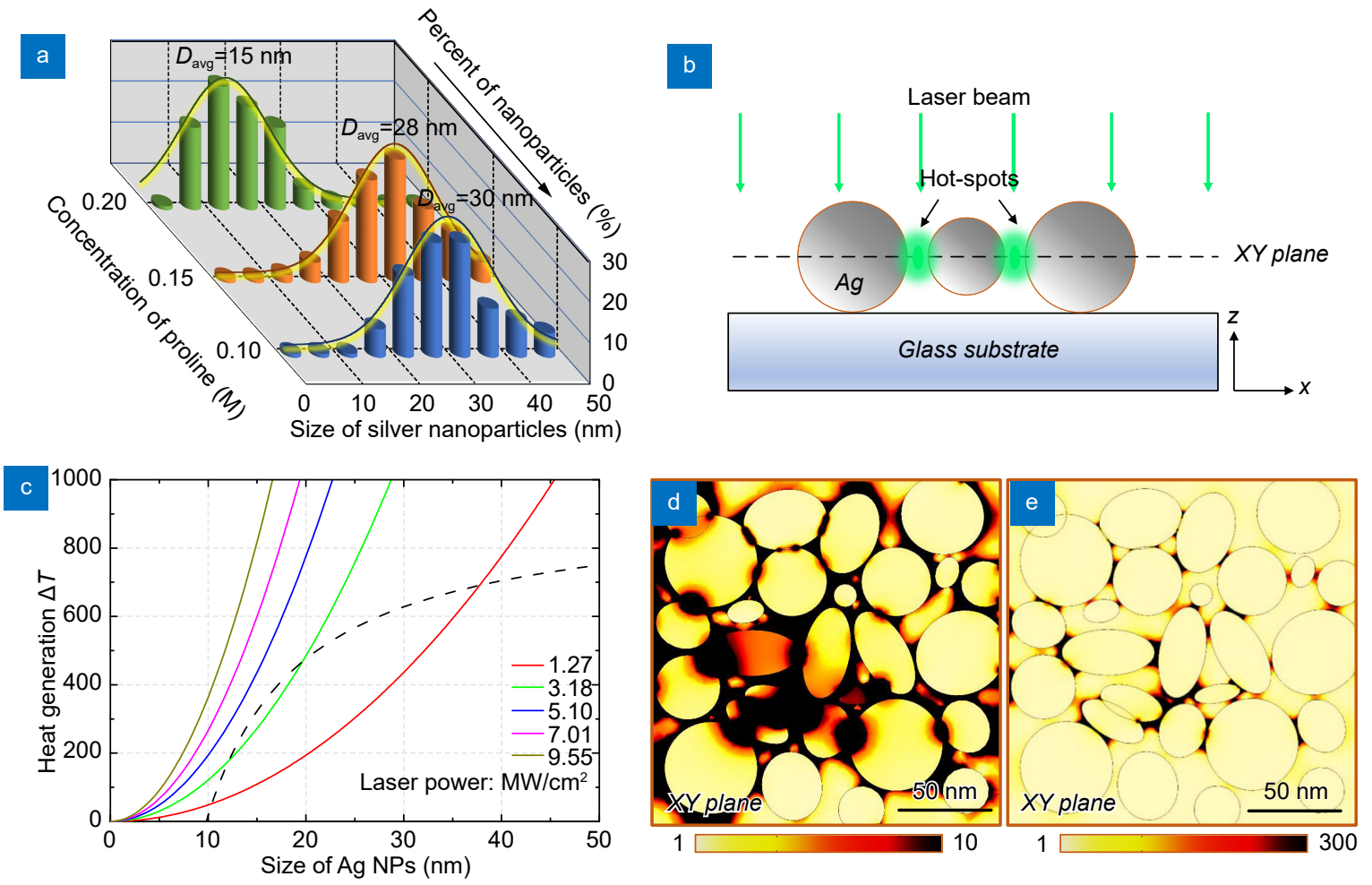

Fig. 5 | (a) Size distribution of Ag NPs in the Ag NWs fabricated in the silver ion contained precursor solutions at different concentrations of surfactant. (b) Schematic of simulation setup, a planar configuration is taken as an example. (c) Calculated temperature increasing $\Delta T\left({ }^{\circ} \mathrm{C}\right)$ at the surface of Ag NPs as a function of Ag NPs size, considering the local light intensity increased 300 times. The black areas (hot-spots) present the simulation results of the temperature distributions when the enhanced light field intensity increases 10 (d) and 300 (e) times by using FEM method.

the electric field and heating intensity are greatly enhanced as shown in Fig. S6. The concentrated light field intensity at the hot-spots (the gaps between adjacent $\mathrm{Ag}$ NPs) has increased hundreds of times compared to the uniform incident light field shown in Figs. 5(d,e) and S6(c-f).

As we mentioned before, Ag NPs with different sizes have their corresponding heat generation $\Delta T$ and melting point $T_{\mathrm{m}}$. We have calculated the temperature increase $\Delta T$ at the surface of Ag NPs with varied diameters of $20 \mathrm{~nm}$ and $40 \mathrm{~nm}$ at different power densities of 1.27, 3.18, 5.10, 7.01 and $9.55 \mathrm{MW} / \mathrm{cm}^{2}$ in Fig. S7. When the SPR effect is not considered, the Ag NPs cannot reach the melting point. Considering the local light intensity being increased 300 times, we have recalculated local temperature increase of $\Delta T$ at the gaps among adjacent Ag NPs as a function of illumination power density as shown in Fig. 5(c). The black dash line presents the critical value of required heat generation to reach the melting point $T_{\mathrm{m}}$ (Fig. S5). The heat generation increases with the laser power density for Ag NPs. Specifically, when the laser power density is relatively low (1.27 and
3.18 MW/ $/ \mathrm{cm}^{2}$ ), the Ag NPs could not be melted completely as illustrated in Fig. 5(c). The Ag NPs with size ranging from $12 \mathrm{~nm}$ to $37 \mathrm{~nm}$ are unable to be melted. All of the Ag NPs ranged from $10 \mathrm{~nm}$ to $50 \mathrm{~nm}$ at the different power densities $\left(I_{0}\right)$ of $5.10,7.01$, and 9.55 $\mathrm{MW} / \mathrm{cm}^{2}$ can be melted completely, resulting in continuous compact Ag NW. As the laser power density continues to increase over $9.55 \mathrm{MW} / \mathrm{cm}^{2}$, the Ag NPs melt more thoroughly and the conductivity of Ag NWs will not be significantly improved. The experimental results shown in Fig. 5(a) suggest that the dependency between the resistance $R$ and the laser power density $I_{0}$ tends to be flat. It is not advised to improve the conductivity by increasing the laser power density. Therefore, a low density of light is insufficient to melt the whole nanoparticles, but if Ag NPs are in contact, they can be welded and melted together by laser irradiation.

The smaller the particles size, the more particleparticle gaps, leading to the bigger resistance value ${ }^{15}$. As shown in Fig. 4(a, b), the resistance value of Ag NWs fabricated from $\mathrm{Ag}$ ions with the proline concentration of $0.20 \mathrm{M}$ (light blue area) is larger than that of $0.10 \mathrm{M}$ 
and $0.15 \mathrm{M}$ (light red area). Furthermore, the more particle-particle gaps, the easier chance to form hot-spots to improve the conductivity. Therefore, the resistance value of Ag NWs containing small NPs decrease rapidly with the increasing power density or nanosoldering time compared to that of large NPs. All of the resistance values gradually decrease as time increases, approaching the saturation state. This is because the soldering as well as melting become faster nonlinearly with the power density or nanosoldering time increasing. In our strategy, laser induced soldering and melting of NPs could be locally controlled in a single Ag NW, in which local heating occurs in the vicinity of the NPs. In addition, a large number of $\mathrm{Ag}$ NWs can be treated simultaneously without the necessary for precise irradiation of the laser beam at an individual Ag NW.the vicinity of the NPs. In addition, a large number of Ag NWs can be treated simultaneously without the necessity for precise irradiation of the laser beam at an individual Ag NW.

\section{Conclusions}

We have obtained the highly conductive silver nanowire electrodes by FsLDW with the assistance of the subsequent laser nanosoldering. Laser nanosoldering of $\mathrm{Ag}$ NPs was utilized to achieve the low temperature and localized nanosoldering based on surface plasmon resonance enhancement photothermal effect. The dependence of resistance on the nanosoldering time and power density of pulse laser has been investigated, which is of critical importance for improving the electric conductivity of Ag NWs. The plasmon-enhanced laser nanosoldering process has successfully enhanced the electric properties of Ag NWs corresponding to a conductivity of $24.51 \times 10^{6}$ $\mathrm{S} / \mathrm{m}$, which is up to $39 \%$ of that of the bulk Ag. Furthermore, we propose the surface plasmon resonance assisted theory to illustrate the nanosoldering process, which is in good agreement with the experimental results. This plasmon-enhanced laser nanosoldering of $\mathrm{Ag}$ NWs can be achieved in a controlled manner to yield high-performance nanoelectrode for the further applications in microelectronics field.

\section{References}

1. Hao YY, Gao J, Xu ZS, Zhang N, Luo J et al. Preparation of silver nanoparticles with hyperbranched polymers as a stabilizer for inkjet printing of flexible circuits. New J Chem 43, 2797-2803 (2019).

2. Xiong WW, Liu HL, Chen YZ, Zheng ML, Zhao YY et al. Highly conductive, air-stable silver nanowire@iongel composite films toward flexible transparent electrodes. Adv Mater 28 , 7167-7172 (2016).

3. Fernandes IJ, Aroche AF, Schuck A, Lamberty P, Peter CR et al. Silver nanoparticle conductive inks: synthesis, characterization, and fabrication of inkjet-printed flexible electrodes. Sci Rep 10, 8878 (2020).

4. Du DX, Yang X, Yang YL, Zhao YZ, Wang YH. Silver nanowire ink for flexible circuit on textiles. Micromachines 10, 42 (2019).

5. Lee S, Jang J, Park T, Park YM, Park JS et al. Electrodeposited silver nanowire transparent conducting electrodes for thinfilm solar cells. ACS Appl Mater Interfaces 12, 6169-6175 (2020).

6. Tang HH, Feng HR, Wang HK, Wan XJ, Liang JJ et al. Highly conducting Mxene-silver nanowire transparent electrodes for flexible organic solar cells. ACS Appl Mater Interfaces 11, 25330-25337 (2019).

7. Lee $\mathrm{J}$, Lee $\mathrm{P}$, Lee $\mathrm{H}$, Lee $\mathrm{D}$, Lee $\mathrm{SS}$ et al. Very long $\mathrm{Ag}$ nanowire synthesis and its application in a highly transparent, conductive and flexible metal electrode touch panel. Nanoscale 4, 6408-6414 (2012).

8. Yang H, Bai SC, Chen TR, Zhang Y, Wang HF et al. Facile fabrication of large-scale silver nanowire-PEDOT: PSS composite flexible transparent electrodes for flexible touch panels. Mater Res Express 6, 086315 (2019).

9. Zhao $Y Y$, Zheng ML, Dong $X Z$, Jin F, Liu J et al. Tailored silver grid as transparent electrodes directly written by femtosecond laser. Appl Phys Lett 108, 221104 (2016).

10. Hirt L, Reiser A, Spolenak R, Zambelli T. Additive manufacturing of metal structures at the micrometer scale. Adv Mater 29, 1604211 (2017).

11. Skylar-Scott M A, Gunasekaran S, Lewis J A. Laser-assisted direct ink writing of planar and 3D metal architectures. Proc Natl Acad Sci USA 113, 6137-6142 (2016).

12. Cheng $T$, Wu $Y W$, Shen $X Q$, Lai $W Y$, Huang $W$. Inkjet printed large-area flexible circuits: a simple methodology for optimizing the printing quality. J Semicond 39, 015001 (2018).

13. Ke SH, Xue QW, Pang CY, Guo PW, Yao WJ et al. Printing the ultra-long $\mathrm{Ag}$ nanowires inks onto the flexible textile substrate for stretchable electronics. Nanomaterials 9, 686 (2019).

14. Cao YY, Takeyasu N, Tanaka T, Duan XM, Kawata S. 3D metallic nanostructure fabrication by surfactant-assisted multiphoton-induced reduction. Small 5, 1144-1148 (2009).

15. Ren $X L$, Zheng ML, Jin F, Zhao $Y Y$, Dong $X Z$ et al. Laser direct writing of silver nanowire with amino acids-assisted multiphoton photoreduction. J Phys Chem C 120, 26532-26538 (2016).

16. Xu BB, Xia H, Niu LG, Zhang YL, Sun K et al. Flexible nanowiring of metal on nonplanar substrates by femtosecond-laser-induced electroless plating. Small 6, 1762-1766 (2010).

17. Wang $\mathrm{H}$, Zhang $\mathrm{YL}$, Xia H, Chen QD, Lee KS et al. Photodynamic assembly of nanoparticles towards designable patterning. Nanoscale Horiz 1, 201-211 (2016).

18. Jia YC, Wang SX, Chen F. Femtosecond laser direct writing of flexibly configured waveguide geometries in optical crystals: fabrication and application. Opto-Electron Adv 3, 190042 (2020).

19. Livakas N, Skoulas E, Stratakis E. Omnidirectional iridescence via cylindrically-polarized femtosecond laser processing. OptoElectron Adv 3, 190035 (2020).

20. Gu WB, Yuan W, Zhong T, Wu XZ, Zhou CS et al. Fast near 
infrared sintering of silver nanoparticle ink and applications for flexible hybrid circuits. RSC Adv 8, 30215-30222 (2018).

21. Han YD, Zhang SM, Jing HY, Wei J, Bu FH et al. The fabrication of highly conductive and flexible $\mathrm{Ag}$ patterning through baking $\mathrm{Ag}$ nanosphere - nanoplate hybrid ink at a low temperature of $100{ }^{\circ} \mathrm{C}$. Nanotechnology 29, 135301 (2018).

22. Kim J, Lin CY, Xing WD, Mecartney ML, Potma EO et al. Laser annealing of nanocrystalline gold nanowires. ACS App/ Mater Interfaces 5, 6808-6814 (2013).

23. Garnett EC, Cai WS, Cha JJ, Mahmood F, Connor ST et al. Self-limited plasmonic welding of silver nanowire junctions. Nat Mater 11, 241-249 (2012).

24. Jahn SF, Blaudeck T, Baumann RR, Jakob A, Ecorchard P et al. Inkjet printing of conductive silver patterns by using the first aqueous particle-free mod ink without additional stabilizing ligands. Chem Mater 22, 3067-3071 (2010).

25. Becerril HA, Mao J, Liu ZF, Stoltenberg RM, Bao ZN et al. Evaluation of solution-processed reduced graphene oxide films as transparent conductors. ACS Nano 2, 463-470 (2008).

26. Wang X, Zhi LJ, Müllen K. Transparent, conductive graphene electrodes for dye-sensitized solar cells. Nano Lett 8, 323-327 (2008).

27. Kumpulainen T, Pekkanen J, Valkama J, Laakso J, Tuokko R et al. Low temperature nanoparticle sintering with continuous wave and pulse lasers. Opt Laser Technol 43, 570-576 (2011).

28. Ko SH, Pan H, Grigoropoulos CP, Luscombe CK, Fréchet JMJ et al. All-inkjet-printed flexible electronics fabrication on a polymer substrate by low-temperature high-resolution selective laser sintering of metal nanoparticles. Nanotechnology 18, 345202 (2007).

29. Chung WH, Jang YR, Hwang YT, Kim SH, Kim HS. The surface plasmonic welding of silver nanowires via intense pulsed light irradiation combined with NIR for flexible transparent conductive films. Nanoscale 12, 17725-17737 (2020).

30. Yang M, Chon MW, Kim JH, Lee SH, Jo JD et al. Mechanical and environmental durability of roll-to-roll printed silver nanoparticle film using a rapid laser annealing process for flexible electronics. Microelectron Reliab 54, 2871-2880 (2014).

31. Huang $H$, Sivayoganathan M, Duley WW, Zhou Y. Efficient localized heating of silver nanoparticles by low-fluence femtosecond laser pulses. App/ Surf Sci 331, 392-398 (2015).

32. Govorov AO, Richardson HH. Generating heat with metal nanoparticles. Nano Today 2, 30-38 (2007).

33. Pitsillides CM, Joe EK, Wei XB, Anderson RR, Lin CP. Selective cell targeting with light-absorbing microparticles and nanoparticles. Biophys J 84, 4023-4032 (2003).

34. Govorov AO, Zhang W, Skeini T, Richardson $\mathrm{H}$, Lee $\mathrm{J}$ et al. Gold nanoparticle ensembles as heaters and actuators: melting and collective plasmon resonances. Nanoscale Res Lett $\mathbf{1}$, 84 (2006).

35. Dittrich S, Barcikowski S, Gökce B. Plasma and nanoparticle shielding during pulsed laser ablation in liquids cause ablation efficiency decrease. Opto-Electron Adv 4, 200072 (2021).

36. Liu ZW, Li Q, Zhang WC, Yang YQ, Qiu M. Nanoscale control of temperature distribution using a plasmonic trimer. Plasmonics 10, 911-918 (2015).
37. Zhang WC, Li Q, Qiu M. A plasmon ruler based on nanoscale photothermal effect. Opt Express 21, 172-181 (2013).

38. Hu AQ, Liu S, Zhao JY, Wen T, Zhang WD et al. Controlling plasmon - exciton interactions through photothermal reshaping. Opto-Electron Adv 3, 190017 (2020).

39. Tseng ML, Huang YW, Hsiao MK, Huang HW, Chen HM et al. Fast fabrication of a $\mathrm{Ag}$ nanostructure substrate using the femtosecond laser for broad-band and tunable plasmonic enhancement. ACS Nano 6, 5190-5197 (2012).

40. Chen Q, Liang L, Zheng Q L, Zhang Y X, Wen L. On-chip readout plasmonic mid-IR gas sensor. Opto-Electron Adv 3, 190040 (2020).

41. Chen X, Chen YT, Yan M, Qiu M. Nanosecond photothermal effects in plasmonic nanostructures. ACS Nano 6, 2550-2557 (2012).

42. Wen L, Chen YF, Liu WW, Su Q, Grant J et al. Enhanced photoelectric and photothermal responses on silicon platform by plasmonic absorber and omni- schottky junction. Laser Photonics Rev 11, 1700059 (2017).

43. Sun $\mathrm{Q}, \mathrm{Yu} \mathrm{H}$, Ueno K, Zu S, Matsuo $\mathrm{Y}$ et al. Revealing the plasmon coupling in gold nanochains directly from the near field. Opto-Electron Adv 2, 180030 (2019).

44. Hu YW, Liang C, Sun XY, Zheng JF, Duan J A et al. Enhancement of the conductivity and uniformity of silver nanowire flexible transparent conductive films by femtosecond laser-induced nanowelding. Nanomaterials 9, 673 (2019).

45. Ding LF, Davidchack RL, Pan JZ. A molecular dynamics study of sintering between nanoparticles. Comput Mater Sci 45, 247-256 (2009)

46. Kim SJ, Jang DJ. Laser-induced nanowelding of gold nanoparticles. Appl Phys Lett 86, 033112 (2005).

47. Mafuné F, Kohno JY, Takeda Y, Kondow T. Nanoscale soldering of metal nanoparticles for construction of higher-order structures. J Am Chem Soc 125, 1686-1687 (2003).

48. Shyjumon I, Gopinadhan M, Ivanova O, Quaas M, Wulff H et al. Structural deformation, melting point and lattice parameter studies of size selected silver clusters. Eur Phys J D 37, 409-415 (2006).

\section{Acknowledgements}

We are grateful for financial supports from the National Key Research and Development Program of China (Grant No. 2016YFA0200502), the National Natural Science Foundation of China (NSFC, Grant Nos. 91323301, 61975213, 61475164 and 51673208), and the Beijing Natural Science Foundation (Grant No. 2182079). Beijing Science and Technology Commission Project (Z161100001516013).

\section{Author contributions}

The manuscript was written through contributions of all authors. All authors have given approval to the final version of the manuscript.

\section{Competing interests}

The authors declare no competing financial interests.

\section{Supplementary information}

Supplementary information for this paper is available at https://doi.org/10.29026/oea.2021.200101 